\section{ARTÍCULO DE REVISIÓN CIENTÍFICA}

DOI: $10.47864 / S E(50) 2020 p 40-53119$
Sociedad Colombiana de la Ciencia del Suelo

\title{
INDICADORES FÍSICOS E HÍDRICOS Y USO DEL SUELO EN LOS FRUTALES
}

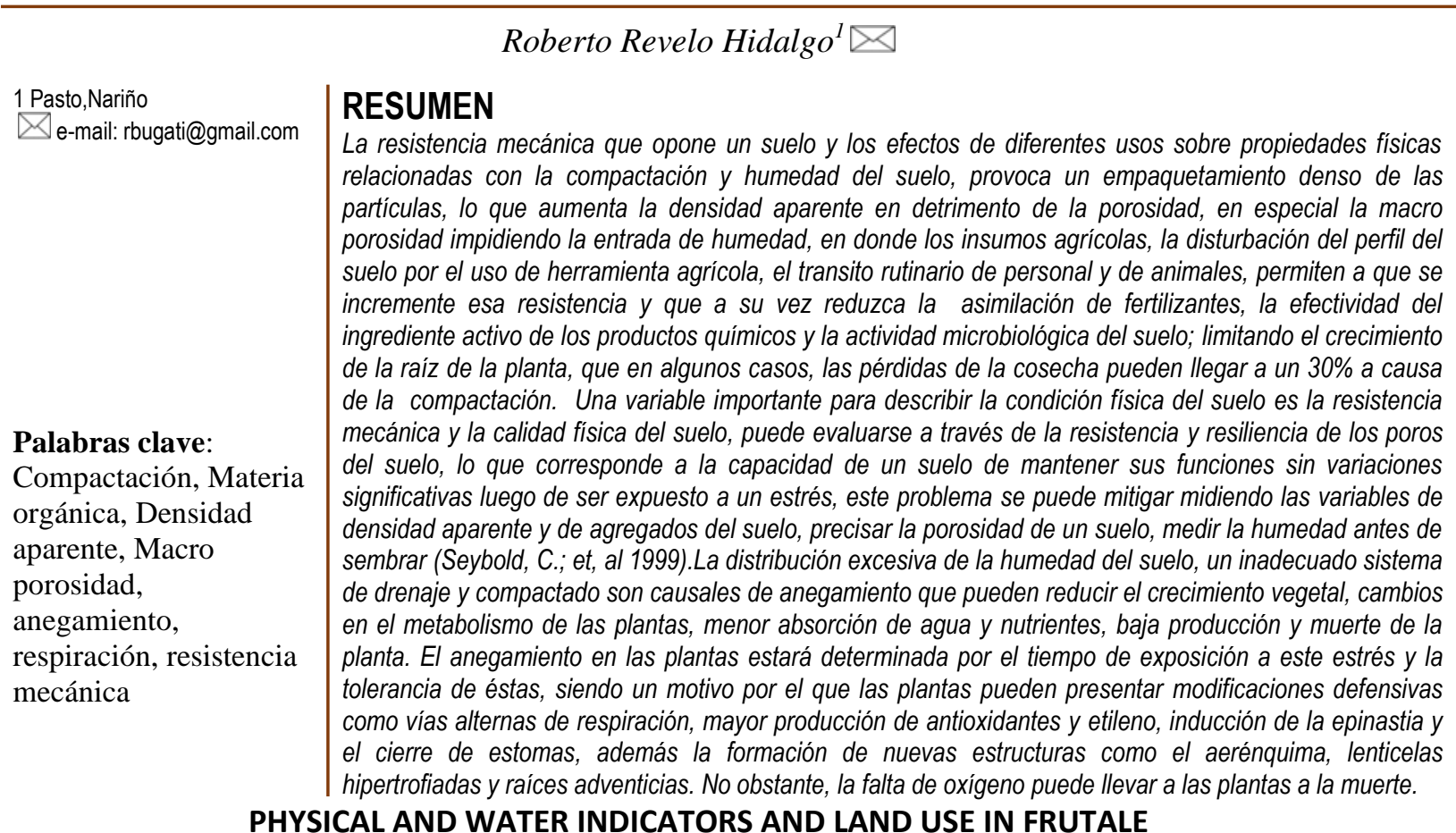

Keywords:

Compaction, Organic matter, Apparent density, Macro porosity, waterlogging, respiration, mechanical resistance

\section{ABSTRACT}

The mechanical resistance that a soil opposes and the effects of different uses on physical properties related to soil compaction and moisture, causes a dense packing of the particles, which increases the apparent density to the detriment of porosity, especially macro porosity preventing the entry of humidity, where agricultural inputs, the disturbance of the soil profile by the use of agricultural tools, the routine transit of personnel and animals, allow that resistance to be increased and that in turn reduce the assimilation of fertilizers, the effectiveness of the active ingredient of chemicals and the microbiological activity of the soil; limiting the growth of the root of the plant, which in some cases, the losses of the crop can reach $30 \%$ due to compaction. An important variable to describe the physical condition of the soil is the mechanical resistance and the physical quality of the soil, it can be evaluated through the resistance and resilience of the soil pores, which corresponds to the capacity of a soil to maintain its functions without significant variations after being exposed to stress, this problem can be mitigated by measuring the variables of apparent density and soil aggregates, specifying the porosity of a soil, measuring the humidity before planting (Seybold, C .; et, al 1999 Excessive distribution of soil moisture, an inadequate drainage system and compaction are causes of waterlogging that can reduce plant growth, changes in plant metabolism, less absorption of water and nutrients, low production and death of the plant. Waterlogging in plants will be determined by the time of exposure to this stress and their tolerance, being one reason why plants can present defensive modifications such as alternate respiratory pathways, greater production of antioxidants and ethylene, induction of epinasty and the closure of stomata, as well as the formation of new structures such as the aerenchyma, hypertrophied lenticels and adventitious roots. However, a lack of oxygen can lead to plants dying. 


\section{INTRODUCCIÓN}

El recurso suelo es esencial para el sostenimiento físico y químico de todos los ecosistemas terrestres. Su degradación es definida como la pérdida a largo plazo en la función y productividad de los ecosistemas, causada por alteraciones, a partir de las cuales el suelo no puede recuperarse sin ayuda (González O. et al., 2009). Los cambios en el uso de la tierra producen importantes modificaciones en la estructura y funcionamiento de los ecosistemas, asociado con la disminución en los contenidos de materia orgánica (MO) se estaría produciendo cambios a nivel de macro porosidad que condicionarían una menor eficiencia de uso del agua, acentuando procesos de degradación física de los suelos. (Cadena B. et al 2012), en Colombia son muchos los errores por uso inadecuado de las metodologías de labranza, generando erosión y detrimento de las propiedades físicas y químicas del suelo al arar y rastrillar el suelo se le pulveriza totalmente, acabando de esta forma la estructura natural favorable del suelo.

(Cadena B. et al 2012), el 100\% de los suelos dedicados a la explotación agropecuaria en Nariño se encuentra en proceso acelerado de degradación por erosión y/o alteración de sus propiedades físicas, químicas y biológicas, el proceso se agrava debido al conflicto persistente de tecnología no apropiada para los suelos de la región y el uso de la labranza tiende a aumentar paulatinamente la densidad y la compactación de los suelos.

El desafío de hoy es enfrentar el actual cambio climático que permita la expresión del potencial productivo de los cultivos, para incrementar la cantidad de alimento producido con el menor impacto ambiental

posible, ya sea en términos de deterioro de la calidad del recurso "suelo" (degradación físico-química) en donde se expone la profundidad máxima que alcanzan las raíces, aunque esté condicionada por las características físicas del suelo y la acción de limitantes como compactación, falta de aireación, altos niveles freáticos, la distribución de la humedad disponible también condiciona la profundidad final del enraizamiento, o de la atmósfera (aumento de la emisión de gases de efecto invernadero, reducción en la captura de carbono, balances negativos de nutrientes, etc). En general, se acepta que el deterioro químico del suelo puede resolverse con prácticas adecuadas de fertilización, mientras que el deterioro físico no está debidamente solucionado.

Las limitantes físicas, como la compactación del suelo es un problema que se puede intensificar por el sistema o técnicas de labranza, el tránsito de la maquinaria, el pisoteo de los animales especialmente cuando la tierra está húmeda y el fuego (Botta et al., 2006), afectando la porosidad, la densidad, la resistencia mecánica, la conductividad hidráulica, la infiltración del agua, el desarrollo radical de las plantas, disminución del intercambio gaseoso necesarios para la formación de sus tejidos. Estas propiedades impactan directa e indirectamente sobre el crecimiento de las plantas y la calidad de los suelos destinados a la producción de alimentos, como consecuencia sobre la productividad y eficiencia de uso del recurso agua y la disponibilidad de los nutrientes en solución del suelo. 
(Singh et al. 2010, Larcher, 2003) El estrés abiótico por anegamiento reduce la calidad de los suelos y la productividad de los cultivos y afecta grandes áreas del mundo estiman que en América Latina el 13.3\% de las tierras cultivables muestran drenaje deficiente debido a la fisiografía que promueve la inundación, niveles freáticos altos o aguas superficiales estancadas. Existe peligro en plantaciones cercanas de ríos grandes y también, de ríos pequeños que repetidamente pasan sus aguas sobre las orillas y que son fenómenos que no ocurren solamente por el cambio climático

Las plantaciones en los valles pueden sufrir el anegamiento durante la temporada de lluvia, especialmente en los casos de un deficiente drenaje, siendo esta situación muy perjudicial en especies como el aguacate (Persea americana), papaya (Carica papaya), lichi (Litchi chinensis) y piña (Ananas comosus), en las cuales el agua estancada origina problemas de severas enfermedades en las raíces. (Paull, 2011, Iacona et al. 2013). Clasifican las plantas como organismos aeróbicos que necesitan la disponibilidad de oxígeno para la absorción de nutrientes por lo cual el agua en exceso alrededor de las raíces puede generar condiciones letales, (Gil, et al 2007) mencionan los efectos más nocivos del anegamiento sobre las plantas leñosas: reducción del crecimiento del tallo y de las raíces, cambios en la toma de nutrientes y la translocación de los carbohidratos, además el aumento de su senescencia y la mortalidad.

(Yetisir et al. 2006, Aldana. 2012, Schaffer 2006), atribuyen esta disminución en crecimiento y productividad de muchos cultivos sensibles al hecho de que en el agua los gases se mueven más despacio causando la deficiencia de oxígeno que es, un elemento crucial para la supervivencia de muchas plantas y microorganismos del suelo, a tal punto, que las pérdidas ocasionadas por este tipo de estrés ambiental, pueden llegar hasta el $40 \%$ del total de la producción.

En contraste a suelos alcalinos, en los cuales el estrés por anegamiento de corta duración puede ser favorable para la nutrición de las plantas, el anegamiento prolongado puede reducir la asimilación neta de dióxido de carbono, la conductancia estomática y la absorción de nutrientes, ocasionando la disminución del crecimiento de tallos y raíces, el marchitamiento y posiblemente, la muerte de las plantas, dependiendo de su nivel de tolerancia que varía entre especies y variedades, además de estar influenciado por la temperatura y las condiciones medioambientales en general (Schaffer, et al. 2006).

La tolerancia al anegamiento no varía solamente entre las variedades sino en gran escala entre los patrones, por lo cual (Schwarz, et al. 2010) recomiendan el uso de los patrones que son capaces de reducir el efecto del estrés externo en un máximo sobre la variedad injertada, y no se usan solamente patrones de la misma especie, sino también de otras, como es el caso del ciruelo para ciertas variedades de duraznero (Orazem, et al. 2011).

A pesar de que existen algunas opciones de manejo tales como incluir gramíneas en las rotaciones, aumentar el contenido de materia orgánica, tránsito controlado, control presión de neumáticos, la des compactación mecánica, entre otras, se requiere mayor estudio para la región. Es de importancia que, el concepto de sostenibilidad de un sistema agrícola esté asociado al manejo que realiza el productor, que es quien tiene y decide por el uso del suelo. Cada sitio se encuentra inmerso en una situación agroclimática que genera 
modificaciones, pero son escasas las acciones que se pueden realizar para modificarla. Aunque, el manejo y sus consecuencias son responsabilidad directa de quien trabaja el suelo. Así, las condiciones edafo climáticas sumado a errores de manejo, pueden agravar el daño.

\section{Factores que permiten el surgimiento $y$ desarrollo de la compactación:}

\subsection{Factores naturales.}

La naturaleza geológica del suelo caracteriza sus propiedades físicas y mecánicas, las cuales influyen en su compresibilidad y compactibilidad, estas dependen de: la textura; estructura; contenido de materia orgánica y humedad del suelo (González, O. et al., 2009); un ambiente favorable para el desarrollo radical significa que el suelo esté, entre otras condiciones, libre de impedancias mecánicas. El término impedancias mecánicas se relaciona con la existencia de capas de suelo con elevadas resistencias, o densidades aparentes, o estructuras desfavorables (masiva o granular), las cuales son capaces de afectar negativamente $\mathrm{y} / \mathrm{o}$ impedir el crecimiento de los cultivos a través de efectos directos (menor emergencia de plántulas; menor crecimiento de raíces) e indirectos (menor absorción de nutrientes). (Álvarez, C.; Fernández, P. 2015).

La compactibilidad aumenta con el contenido de humedad. La humedad del suelo es el factor que mayor influencia tiene en la compactación (González, O. et al., 2009). Durante la aplicación de presiones al suelo, a medida que esta aumenta hasta alcanzar la humedad crítica de compactación mayor compactación se provoca; a este valor de humedad la densidad de volumen del suelo alcanza el máximo, para una presión de compactación dada, igualmente influye la fuerza de gravedad, la lluvia y los ciclos de humedecimiento y secado del suelo. El humedecimiento favorece una disminución de la densidad de volumen, debido a que la presencia de agua en los poros amortigua y dificulta el acercamiento de las partículas del mismo, estos se expanden y disminuye el efecto de la compactación, aunque no siempre es perjudicial; durante la siembra es necesario una cierta compactación del suelo para que la semilla tenga un contacto adecuado con este, facilitando el acceso de la raíz al aire y nutrientes del suelo.

El contenido de materia orgánica la compactibilidad está influenciada no solo por su contenido, sino por el tipo de materia orgánica, ya que material parcialmente descompuesto y altamente humidificado incrementa la resistencia del suelo a la compactación.

\subsection{Factores antropogénicos.}

El principal efecto que ejercen las fuerzas de compresión sobre el suelo es el cambio en la porosidad, especialmente una variación en la distribución de su tamaño (Gutiérrez, F. 2014). A medida que las fuerzas de compresión aumentan, los poros grandes se colapsan. En otras palabras, un incremento en la densidad del suelo implica una reducción del espacio poroso. Dada la relación directa que tiene la macro-porosidad con el crecimiento de las raíces, es importante considerar el volumen total ocupado por macro poros que superan los 0,1 $\mathrm{mm}$ de diámetro. A medida que disminuye el volumen de poros que superan 
este diámetro, se afecta el desarrollo de las raíces del cultivo.

Los suelos bajo producción agrícola y/o ganadera están expuestos a la aplicación de fuerzas de estrés debido al tránsito de maquinaria o de animales. Los sistemas agrícolas, e incluso los sistemas mixtos, se manejan en una alta proporción bajo siembra directa (SD), siendo el tránsito de maquinarias y el pisoteo animal los factores que conducen a la compactación de los suelos. La presencia de pisos de arado y el encostramiento superficial de los suelos asociados a la labranza convencional (LC). En el sistema de SD se pueden distinguir dos zonas o profundidades de compactación: 1) una que se ubica más superficialmente, la cual en sistemas laboreados es borrada por la labranza, y 2) otra más profunda en el perfil, presente en cualquier tipo de sistema de labranza.

La compactación superficial está más asociada a la presión que ejerce el tránsito y la consolidación de las partículas, mientras que la compactación profunda se relaciona más con el peso del agente que aplica el estrés. En SD, observaron un leve aumento de la densidad aparente y un importante aumento de la resistencia a la penetración. (Álvarez, C.; Fernández, P. 2015) evaluaron la estructura del suelo a través del método de perfil cultural, en un sistema bajo 7 años de agricultura en SD observaron una capa superficial, de $3 \mathrm{~cm}$ de espesor, de tierra fina y restos vegetales, con una estructura favorable con terrones individualizados presentándose luego una capa continua de 4-6 cm de espesor con estructuras laminares, de baja porosidad y caras de ruptura lisas.
Cuando el tránsito se realiza en condiciones de suelo húmedo, el resultado negativo se verá maximizado y la profundidad de la zona afectada será mayor (Balbuena, R. 2000). La compactación de la capa superficial está relacionada con la presión sobre el terreno, mientras que la compactación a mayores profundidades está relacionada con el peso sobre los sistemas de rodaje determinando este la intensidad a la cual estas presiones decrecen con la profundidad (González O. et al., 2009). A medida que se incrementa la presión de inflado del neumático menor es el área de contacto debido a una mayor rigidez del mismo, lo que origina un aumento de la deformación del suelo y de la densidad de volumen, transmitiéndose mayores tensiones y a más profundidad (Marsili, et al., 1998). Las tensiones que se originan en la capa superficial están relacionadas con la presión de inflado, aunque en el subsuelo el efecto de la presión de inflado no es significativo.

\section{La densidad aparente (Da) y la resistencia mecánica $(\mathbf{R m})$}

(Arun. 2012). La humedad en exceso sobre lubrica el suelo y lo hace inestable, cambios estructurales por compactación alteran las propiedades volumétricas del suelo, lo que repercute en el desarrollo y crecimiento de las plantas porque decrece la conductividad hidráulica y la capacidad de retención de agua y se altera la difusión de gases en su interior. Estos cambios provocan una deficiente aireación y un suministro inadecuado de oxígeno para el desarrollo de las plantas. Así mismo, la degradación de la estructura del suelo por compactación provoca una ralentización o paralización del desarrollo de las raíces, al aumentar la Rm. 
Los valores críticos de impedancia mecánica del suelo son variables y dependen del tipo de planta y de las características y propiedades del medio edáfico. Con valores de impedancia mecánica superiores a $2 \mathrm{MPa}$ se reduce significativamente el crecimiento de las raíces.

La DA y la $\mathrm{Rm}$ son propiedades usadas para analizar la degradación del suelo por compactación debido a las actividades agropecuarias. La relación entre ambas variables ha originado a resultados contrastantes. (Sojka, et al. 2002; Sepúlveda.2009) señalan que cada una presenta una diferente sensibilidad para medir la compactación del suelo $\mathrm{y}$, por tanto, no hay buena correlación entre ellas. (Arun, et al 2012) indica que la $\mathrm{Rm}$ es sensible a los cambios producidos en el suelo por micro grietas, planos de fractura y porosidad; mientras que, la DA es sensible sólo a los cambios en la porosidad del suelo. A una misma densidad del suelo y un mismo contenido de humedad, el suelo es más compresivo cuanto mayor es el contenido de arcilla y menor el contenido de materia orgánica.

Los suelos arenosos presentan mayor fricción entre las partículas lo que dificulta el movimiento de las partículas para posiciones de mayor proximidad. La estrecha relación entre la plasticidad y la consistencia del suelo, es una cualidad que suele ser usada para caracterizar el comportamiento mecánico del suelo, y coincide con los resultados obtenidos por quienes indican que la plasticidad es una variable importante para predecir la susceptibilidad a la compactación del suelo. (Sepúlveda. 2009), expone que el efecto de la materia orgánica sobre la resistencia del suelo puede ser relacionada a el efecto determinante en la formación estabilización de agregados del suelo y considera que la acumulación de materia orgánica puede ser relacionada a dos efectos contradictorios sobre la resistencia del suelo, que son el aumento de la fuerza de ligación entre las partículas minerales y el cambio en el arreglo de las partículas del suelo.

\section{Efectos de la compactación}

El aumento de la resistencia mecánica del suelo va a restringir el crecimiento de las raíces a espacios de menor resistencia, tales como los que se ubican entre las estructuras (terrones), en cavidades formadas por la fauna del suelo (lombrices) y en espacios que se producen por la descomposición de restos orgánicos gruesos (raíces muertas). Esta situación va a producir un patrón de crecimiento característico de raíces aplanadas, ubicadas en fisuras del suelo, con una escasa exploración del volumen total del suelo (Ramírez, R.; Salazar, C. 2012). A medida que la textura se hace más gruesa la densidad aparente presenta un valor crítico más alto. Este hecho es lógico porque la macro porosidad, que es la más afectada por el fenómeno de compactación, se ve menos influida por la disminución general de porosidad a medida que la textura se hace más arenosa y el dominio de los poros gruesos es más amplio.

Se ha demostrado que la compactación del suelo es dañina, cuando ocurre en los primeros $50 \mathrm{~cm}$ de la superficie del suelo, donde ocurre el mayor crecimiento radical de las plantas. Cuando el suelo es compactado, se reduce o se destruye el sistema de macro poros presente en el suelo. Los macro poros son importantes para el movimiento del agua y el aire en el suelo y sin ellos se presentan condiciones 
anaeróbicas en la etapa de crecimiento. Al ocurrir condiciones anaeróbicas en el suelo, se reduce el oxígeno disponible, trae como consecuencia reducción en la des nitrificación, pérdida de nutrimentos en las raíces y cambios en el metabolismo de las plantas. La compactación de los suelos es un problema de generación, ya que se puede agravar y reflejar en la producción, debido a que las prácticas de labranza provocan que aumenten sus niveles, esto se evidencian en la reducción de sus espacios porosos, al formarse una capa dura conocida como piso de arado, lo que da como resultado el desarrollo deficiente de raíces, poco crecimiento de plantas y baja producción (Ramírez, R.; Salazar, C. 2012).

El contenido de agua es un factor muy asociado al grado de compactación de los suelos. Los suelos en estado natural presentan más o menos resistencia de acuerdo a la mayor o menor presencia de humedad, fenómeno que se agudiza en los Vertisoles donde, por su composición montmorrillonítica, ocurren procesos tales de dilatación - contracción que provocan agrietamientos en la capa superficial durante los períodos prolongados de sequía (Ramírez, R.; Salazar, C. 2012)

\section{Compactación de suelos por el pisoteo}

Las principales fuerzas de compactación son la lluvia, la maquinaria agrícola y la carga animal. La lluvia desintegra los agregados, dispersa la arcilla y favorece la compactación superficial, aumentando la densidad aparente de los primeros centímetros de suelo este fenómeno está asociado con un bajo contenido de materia orgánica y altos contenidos de limo y sodio. El efecto de la carga animal se concentra en los primeros cinco centímetros de suelo y se debe al peso del animal en relación a la superficie de contacto con el suelo $(4,0 \mathrm{~kg}$ $\mathrm{cm}^{2}$ ) en el que se posa. (Agüero. J.; Alvarado. A. 1983). La maquinaria agrícola compacta el suelo a mayor profundidad que la lluvia y la carga animal. El arado deja una capa superficial suelta y un sub suelo denso debido a que aplica una presión que oscila entre 0,76 y $0,95 \mathrm{~kg} \mathrm{~cm}^{2}$. Este efecto se acentúa cuando la labranza de tierras se hace a la misma profundidad durante varios años.

En terrenos compactados sembrados de algodón, la raíz pivotante crecía en forma horizontal o se bifurca como "pata de gallina". En áreas ganaderas encontraron la compactación más alta; los valores de compactación en suelos dedicados a ganadería casi duplican los encontrados en suelos de cultivo. En suelos cultivados la resistencia es baja en la superficie, debido al paso de implementos agrícolas y la actividad radical; aumenta hasta cierta profundidad (piso de arado) y decrece a mayor profundidad, en forma similar a lo encontrado en otros lugares. En suelos dedicados a la ganadería el nivel máximo de compactación se encuentra en la superficie, sitio más disturbado por el ganado. (Agüero. J.; Alvarado. A. 1983). La compresión consiste en la disminución del volumen del suelo por la aplicación de una carga alta.

Cuando este proceso ocurre en suelos saturados se denomina consolidación, y compactación cuando ocurre en suelos no saturados. En el primer caso se excluye el agua de los espacios vacíos de la matriz del 
suelo y en el segundo se excluye el aire (Pinzón, A.; Amézquita, E. 1991), considera que la compactación reduce el volumen de poros de mayor diámetro del suelo, causando cambios en el contenido de humedad y en el intercambio de gases entre el suelo y la atmósfera, e impidiendo además el desarrollo de las raíces. El establecimiento de pasturas en el bosque húmedo tropical, junto con el pisoteo de los animales, favorecen la compactación en el horizonte superior del suelo; aunque, se sabe muy poco sobre los cambios físicos y pedogenéticos que estas prácticas ocasionan en el suelo.

El pisoteo de los animales compacta el suelo en los primeros $15 \mathrm{~cm}$, ocasionando una severa disminución en el movimiento interno del agua, y un aumento en la DA; esto trae como consecuencia una disminución en la porosidad y cambios desfavorables en la relación suelo-agua-aire que afectan el desarrollo de las raíces de las plantas y su productividad. Los resultados permiten recomendar que en las zonas de lomerío y de terrazas bajas del piedemonte amazónico de Colombia, la explotación ganadera debe hacerse con cargas animales adecuadas, y en pasturas con especies mejoradas que favorezcan la estructura del suelo. (Pinzón, A.; Amézquita, E. 1991, Medina, C. 2016) establece que la compactación del suelo se puede desarrollar no sólo en las grandes fincas mecanizadas, sino también por actividades de la labranza manual, y por las acciones del pastoreo y pisoteo animal.

La cría de ganado, específicamente vacuno, ha dejado una devastadora y aún no calculada pérdida de las condiciones de páramo, reflejado, especialmente, en la capacidad de retención de agua en el suelo que se ve afectada por el pisoteo constante del ganado, compactándolo y quitándole espacio poroso para acumular el agua. La pérdida de agua causa un reacomodamiento de las unidades estructurales, produciendo modificaciones en los espacios vacíos y el suelo pierde su capacidad de infiltración, dificultando el flujo de agua y la toma de nutrientes de las plantas (Estupiñán. L.; et al 2009). El suelo del páramo, por su alto contenido de materia orgánica, es suelto y suave, aspecto que facilita la compactación causada por el peso de los animales.

Además, las deyecciones del ganado están relacionadas con un aumento de la concentración de carbono en la capa arable y un aumento en el espesor de la capa orgánica; su mineralización presenta problemas como el exceso de nitrógeno, que afecta las características físicoquímicas de los suelos y su capacidad para mantener a los cultivos, sumado los potenciales peligros de contaminación de acuíferos y cursos de agua.

\section{Compactación de los suelos frutícolas}

Los sellamientos superficiales de suelos se han vuelto un problema a nivel mundial en la fruticultura a causa de diversos factores. Entre estos, se encuentran el uso intensivo de maquinaria agrícola en las distintas tareas de manejo de los huertos frutales, como son las aplicaciones sanitarias y equipos de cosecha entre otros, sumado a la incorporación de suelos marginales con problemas de salinidad. Además, la práctica cero labranza en no pocas explotaciones frutales, ha contribuido a estos sellamientos superficiales, al no existir una cubierta vegetal que mejore las características físicas del suelo facilitando su permeabilidad. 
(Peralta, J.; Carrasco, J. 1990), según el INIA realizada una encuesta a fruticultores chilenos el $76 \%$ de los predios encuestados la eficiencia de riego es baja debido, fundamentalmente, al problema de compactación de suelos, que se ha traducido en menor desarrollo de plantas, y en consecuencia la calidad y productividad total ha disminuido.

La compactación de suelo corresponde, en primer término, a estratos endurecidos ubicados sub superficialmente que se forman a consecuencia del uso de herramientas de labranza en forma inadecuada, por ejemplo, sobre suelos con altos contenidos de humedad, o la ejecución de labores siempre a la misma profundidad de trabajo.

También sucede que los implementos utilizados para ciertas labores no son los más apropiados, estas situaciones son especialmente válidas en suelos con hortalizas. El efecto es más crítico en suelos con mayores contenidos de arcilla y mayor grado de humedad. El resultado es la formación de una o más capas endurecidas que impiden el paso del agua y el crecimiento de las raíces hacia zonas más profundas. Este efecto se manifiesta fuertemente en suelos con frutales. El movimiento del agua en el suelo, se ha observado que el flujo en los poros grandes es más afectado por la compactación que el flujo en los poros pequeños. Las propiedades hidráulicas como la infiltración básica y la conductividad hidráulica saturada son afectadas en gran medida por los fenómenos de compactación. (Draghi, L.; et al 2014).

\section{Efectos del anegamiento sobre el crecimiento de los frutales}

(González et al. 2006), manifiestan que las consecuencias que puede ocasionar el anegamiento en cultivo y para el productor son el menor porcentaje de germinación, la disminución del desarrollo vegetativo y del número y tamaño de los frutos, así como el aumento en los niveles de plagas $\mathrm{y}$ enfermedades que finalmente causan los bajos rendimientos hasta la pérdida total de la cosechas, además de los sobrecostos que se derivan de esa situación. El crecimiento de las plantas frutales y la calidad de los frutos pueden ser afectados por el anormal desarrollo de las raíces ya que se modifica el traslado del agua y nutrientes a la parte aérea, siendo más grave cuando las plantas tienen raíces de crecimiento superficial, como sucede en el caso de las plantas de duraznero que requieren de suelos sin excesos de humedad y buen drenaje.

Cuando el suelo es inundando el agua ocupa los poros causando de inmediato una deficiencia del oxígeno y el poco oxígeno en el suelo está consumido por las raíces y los microorganismos en pocas horas. Los problemas que puede llevar consigo el anegamiento comienzan por los cambios a nivel del suelo como es la variación de la acidez, las relaciones entre cationes y la reducción del hierro, conllevando a deficiencias nutricionales, producción de sustancias tóxicas, además escaso o nulo contenido de oxígeno en este medio.

Existen diferencias en la tolerancia de las especies y, en menor grado, entre variedades al anegamiento (Ezin, et al. 
2010) y esta situación puede ser ocasionada en parte porque los requerimientos de oxígeno de la raíz varían mucho entre cultivos y son mayores a medida que la temperatura del suelo y del agua aumentan, debido a que la solubilidad del oxígeno disminuye.

(Ismail, S. et al 2007, Fischer, G. 2012) reportan que el aguacate, al igual que el duraznero (Prunus

persica), exige un $15 \%$ de $\mathrm{O} 2$ en la solución del suelo, mientras que otros, como el manzano

(Malus domestica) y el peral (Pyrus communis), aguantan hasta solamente un 5\%. Especies como las pasifloráceas, especialmente la gulupa (Passiflora edulis Sims), son muy sensibles al anegamiento (Fischer et al. 2009), igualmente como las mencionadas aguacate, papaya, lichi y la piña (Paull, 2011), mientras que el cerezo (Prunus avium), ciruelo (Prunus doméstica y P. salicina) y los cítricos (Citrus spp.) se clasifican como tolerantes intermedios a esta adversidad.

La falta de tolerancia del aguacate a la asfixia radical es debida a la carencia de pelos absorbentes

(Schaffer, 2006, Gil et al. 2007), clasifican algunos frutales, con respecto a la diferencia entre variedades, en un estudio en La Florida, encontró que la variedad de aguacate 'Beta', es más susceptible al anegamiento que la 'Hass' debido a su mayor pérdida de biomasa foliar y radical, al ser sometida a este tipo de estrés.

\section{Adaptaciones fisiológicas}

Entre las adaptaciones fisiológicas a la hipoxia, se encuentra la inducción de la epinastia, con la que se reduce la transpiración debido a que se le confiere protección a las hojas contra el calor y el sol directo como sucede en el caso de las plantas de aguacate (Schaffer et al. 2006). En diferentes cultivos como por ejemplo en los arboles de limón, el cierre estomático puede ser uno de las reacciones al estrés por anegamiento, muy probablemente, para contrarrestar la pérdida de agua, como lo interpretan muchos investigadores del tema.

Una de las adaptaciones más importantes a la inundación es la capacidad de mantener la actividad fotosintética, así como la capacidad de almacenar azucares solubles para las raíces, ya que aunque una planta puede tener altas reservas de azúcares, también debe disponer de una eficiente vía glicolítica que le permita utilizarlos (Parent et al. 2008). Como otro de los mecanismos de adaptación debido a la falta de oxígeno para las raíces, las plantas pueden modificar su metabolismo, haciendo el cambio de su respiración normal a la de la vía de la glicolisis (Jiménez et al. 2012). En condiciones de anoxia, la síntesis de etileno es bloqueada, a diferencia de lo que sucede en condiciones de hipoxia, en las que por el contrario se incrementa promoviendo la formación del aerénquima, además de influir en el crecimiento y desarrollo de la raíz, y darle paso a la epinastia en las hojas.

(Parent et al. 2008), otra de las repuestas de las plantas cuando tienen que padecer el estrés por anegamiento es la promoción de la síntesis de las proteínas del estrés anaeróbico que se producen por inducción de un medio deficiente en oxígeno. Por medio de procesos metabólicos, las casi 20 proteínas del estrés anaeróbico, sintetizadas de manera selectiva en condiciones desfavorables para la producción de energía aeróbica, tienen la capacidad de generar energía independiente de oxígeno

\section{Adaptaciones morfológicas y anatómicas}


La reducción del área foliar es un mecanismo de respuesta que presentan frente al estrés por inundación las plantas, por ejemplo en la uva que se pueden recuperar cuando el estrés es de corta duración, exceptuando que se presente al acercarse la época de cosecha por los daños que se pueden presentar en el producto final (Herralde et al. 2005). El aerénquima es un conjunto de canales que conectan las partes aireadas de la planta con las que están sumergidas para permitirles la llegada de oxígeno (Yetisir et al. 2006), es un tejido interconectado se forma a través de la separación de las células durante el desarrollo y muerte programada de las células lo que puede ocurrir frecuentemente, también en plantas que crecen bajo condiciones normales.

En algunos patrones de peral, la acumulación de la auxina ácido indolacético en la base de las plantas, en conjunto con la síntesis de etileno, son factores que fomentan la aparición de las raíces adventicias.

(Schaffer 2006, Parent et al. 2008), atribuyen al desarrollo de las raíces adventicias una adaptación morfológica importante con el que las plantas pueden mitigar los daños generados por el anegamiento debido a su capacidad de una mayor absorción del O2, reportan que plantas leñosas pueden absorber hasta $90 \%$ más agua a través de sus raíces adventicias comparado con aquellas en las que se removieron estas raíces, encontraron en plantas de anón (Annona glabra) anegadas no solamente el desarrollo de raíces adventicias sino también la formación de lenticelas hipertrofiadas en raíces y el tronco.

Con la intención de favorecer la difusión del oxígeno y la salida de sustancias toxicas producidas por el metabolismo anaeróbico, es posible que se formen abultamientos de tejidos en la base de los tallos resultantes de la división y expansión celular radial, como una adaptación a la falta de oxígeno que se denominan lenticelas hipertrofiadas. (Zude, 2000) en mango, una especie moderadamente resistente al anegamiento la formación de lenticelas hipertrofiadas ha ocurrido en diferentes plantas como las que han estado sometidas a estrés por anegamiento, siendo al parecer uno de los mecanismos que utilizan las plantas para sobrevivir porque facilita la difusión de oxígeno, aunque también se cree que pueden aparecer más por motivo de la temperatura, y no se conoce todavía como participan en la eliminación de metabolitos tóxicos caracterizan el desarrollo de lenticelas hipertrofiadas en plantas de mango como un parámetro para la selección de variedades resistentes que en ocasiones deben ser propagadas clonalmente, por medio de técnicas de cultivo de tejidos teniendo en cuenta que existen variedades que poseen un embrión

zigótico y no sirven para ser usadas como semilla.

(Larcher, 2003) menciona que plantas que crecen en suelos compactos y pobremente aireados pueden desarrollar un sistema de raíces laterales cerca de la superficie del suelo para obtener más oxígeno. La formación de una capa de células lignificadas al interior de la hipodermis y una hipodermis suberizada son adaptaciones con que las plantas consiguen minimizar la pérdida de oxígeno, además de los daños que les puede ocasionar (Rutto et al. 2001) observaron en plántulas de duraznero anegadas que la inoculación de micorriza Gigaspora margarita favoreció un mayor porcentaje de raíces viables, concentraciones del tallo más altas de $\mathrm{P}, \mathrm{K}$ 
y Zn y biomasa, mientras que las plántulas no inoculadas acumularon un contenido más alto de etanol en las raíces.

En citrus (Citrus junos) la colonización con la micorriza, Diversispora spurca, aumentó el crecimiento (altura de la planta, biomasa, longitud y volumen de raíces) comparado con plántulas no inoculadas, por lo cual sugirieron que D. spurca alivia los efectos del anegamiento por su acción sobre la arquitectura radicular y las actividades de enzimas antioxidantes.

\section{REFERENCIAS}

AGÜERO. J.; ALVARADO. A. Compactación y compactabilidad de suelos agrícolas y ganaderos de Guanacaste, Costa Rica. Agronomía Costarricense. 7: 27-33. 1983

ALDANA, F.; GARCÍA, P. GERHARD, F. Efecto del estrés por anegamiento sobre el crecimiento, desarrollo y fisiología de uchuva (Physalis peruviana L.) Universidad Nacional de Colombia. Rev.

Acad. Colomb. Cienc. Ex. Fis. Nat. Vol. 38 Núm. 149. 2014

ALVAREZ, C.; FERNANDEZ, P. La Compactación de los Suelos bajo Agricultura. Facultad de Agronomía, UBA. 13 p. 2015

ARUN. K; YING, C.; MOHAMMAD. S, SHAFIQUR, R. Índice de cono del suelo en relación con la textura del suelo, el contenido de humedad y la densidad aparente para labranza cero y labranza convencional. v. 14, No 1.2012

BALBUENA, R. Compactación del suelo durante la cosecha forestal evolución de las propiedades físicas. Revista Brasileira de
Engenharia Agrícola e Ambiental, v.4, n.3, p.453-459. 2000

BOTTA, G.; D. JORAJURIA; L. DRAGHI: "Influence of the axle load, tire size and configuration, on the compaction of a freshly tilled clayey soil", Journal of Terramechanics, 39: 47-54, 2006.

CADENA, B.; DAVID, B.; RUIZ, H.; MOSQUERA, J.; BENAVIDES, O. Efecto de cinco sistemas de labranza en la erosión de un suelo Vitric Haplustand, bajo cultivo de papa. Facultad Ciencias agrícolas UDENAR. 29p. 2012

DRAGHI, L.; PALANCAR, T.; SORACCO, G.; LOZANO, L.; JORAJURIA, D. Siembra directa y sustentabilidad impacto físico-mecánico sobre el suelo. CONICET. 7 p. 2014

ESTUPIÑÁN. L.; GÓMEZ. J.; BARRANTES. J.; LIMAS. L. Efecto de actividades agropecuarias en las características del suelo en el páramo el granizo, (Cundinamarca - Colombia). Rev. U.D.C.A 12 (2): 79-89. 2009

EZIN, V.; DE LA PENA, R.; AHANCHEDE, A. Flooding tolerance of tomato genotypes during vegetative and reproductive stages. Brazilian Journal of Plant Physiology. V. 22(2). 2010

FISCHER, G.; CASIERRA, F.; PIEDRAHÍTA, W. Eco fisiología de las especies pasifloráceas cultivadas en Colombia. Sociedad Colombiana de Ciencias Hortícolas. p 45-67. 2009

GIL, P.; SCHAFFER, B.; GUTIERREZ, S.; LI, C. Efectos del anegamiento en el estatus hídrico e intercambio gaseoso y biomasa del palto (Persea americana Mill.). En: Proceedings VI World 
Avocado Congress. 13p. 2007

GONZÁlEZ, O.; IGLESIAS, C.; HERRERA, M. Análisis de los factores que provocan compactación del suelo agrícola. Revista Ciencias Técnicas Agropecuarias 18(2), 57-63.2009

GUTIERREZ, F.; Compactación mecánica en suelos Vertisol. UAEM. 16. 2014

HERRALDE，F.; SAVÉ, R.; BIEL，C. Ecophysiological response to flood of seven grapevine cultivars.

Acta Horticulture 689: 137-144. 2005

IACONA, C.; CIRILLI, M.; ZEGA, A.; FRIONI, E.; SILVESTRI, C; MULEO, R. A somaclonal myrobalan rootstock increases waterlogging tolerance to peach cultivar in controlled conditions.

Scientia Horticulturae 156: 1-8. 2013

ISMAIL, S.; OZAWA, K.; KHONDAKER, $\mathrm{N}$. Effect of irrigation frequency and timing on tomato yield, soil water dynamics and water use efficiency under drip irrigation. Eleventh International Water

Technology Conference. 16p. 2007

JIMÉNEZ, J.; MORENO, L.; MAGNITSKIY, S. Respuesta de las plantas a estrés por inundación.

Revista Colombiana de Ciencias Hortícolas. 6(1): 96-109. 2012

LARCHER, W. Physiological plant ecology. Ecophysiology and stress physiology of functional groups. 2003.

MARSILI, A.; SERVADIO, P.; VIGNOZZIB, N. Changes of some physical properties of a clay soil following passage of rubber- and metal-tracked tractors. Soil and Tillage Research. p. 185-199. 1998
MEDINA, M. Efectos de la compactación de suelos por el pisoteo de animales, en la productividad de los suelos. Revista Colombiana Ciencia Animal 8(1):88-93. 2016

ORAZEM, P.; STAMPAR, F.; HUDINA, M. Quality analysis of Redhaven peach fruit grafted on 11 rootstocks of different genetic origin in a replant soil. Food Chemistry 124(4):1691-1698. 2011

PARENT, C.; CAPELLI, N.; BERGER, A.; CREVECOEUR, M.; DAT, J. An overview of plant responses to soil waterlogging. Plant Stress 2(1): 20-27. 2008

PAULL, R.; DUARTE, O. Tropical fruits. Vol. 1. CAB International. 2011

PERALTA, J.; CARRASCO, J. La compactación de los suelos frutícolas $\mathrm{y}$ hortícolas. IPA La Platina n. 60.1990

PINZÓN, A.; AMÉZQUITA, E. Compactación de suelos por el pisoteo de animales en pastoreo en el piedemonte amazónico de Colombia. Pasturas Tropicales, Vol. 13 n. 2. p. 21-26. 1991

RAMÍREZ, R.; SALAZAR, C. Cambios de la resistencia a la penetración en un suelo con diferentes sistemas de manejo y su relación con algunas propiedades físicas en un andisol-marinilla la montañita. 22p. 2012

RUTTO, K.; MIZUTANI, F.; KADOYA, $\mathrm{K}$. Effect of root-zone flooding on micorrizal and nonmycorrhizal peach (Prunus persica Batsch) seedlings. Scientia Horticulturae 94: 285-295. 2001

SCHAFFER, B. Effects of soil oxygen deficiency on avocado (Persea Americana Mill.) trees. En: 
Manejo del Riego y Suelo en el Cultivo del Palto, INIA. p 1-12. 2006

SCHWARZ, D.; ROUPHAEL, Y.; COLLA, G.; VENEMA, J. Grafting as a tool to improve tolerance of vegetables to abiotic stresses: Thermal stress, water stress and organic pollutants Scientia.

Horticulturae 127: 162-171. 2010

SEPÚLVEDA, R. La relación entre la densidad aparente y la resistencia mecánica como indicadores de la compactación del suelo. Agrociencia vol.43 no.3. 2009

SEYBOLD, C.; HERRICK, J.; BREJDA, J. Soil resilience: a fundamental component of soil quality soil science. v. 164. p. 224-234. 1999

SINGH, B.; JOSEPH S.; CAMPS, M.; LIN Y.; MUNROE, P.; CHIA, C.; HOOK, J; VAN ZWIETEN L.;

KIMBER. S; COWIE, A; LEHMANN. J.; FOIDL. N.; SMERNIK. R.; AMONETTE. J. An investigation into the reactions of biochar in soil. Australian Journal of Soil Research 48, 501-515. 2010

SOJKA, R.; LENTZ, D.; ROBBINS, W.; KINCAID, D.; WESTERMANN, D. Polyacrylamide for surface irrigation to increase nutrient-use efficiency and protect water quality. Communications in Soil

Science and Plant Analysis, 32(7-8):1203$1220 \cdot 2001$

YETISIR, H.; CALISKAN M.; SOYLU, S.; SAKAR, M. Some physiological and growth responses of watermelon Citrullus lanatus (Thunb.) Matsum and Nakai grafted onto Lagenaria siceraria to flooding. Environmental and Experimental Botany 58: 1-8. 2006

ZUDE S.; M. SCHAFFER, B. Influence of soil depletion on iron uptake and reduction in mango

(Mangifera indica L.) roots. Horticultural Society 113: 1-4. 2000 
